\title{
EFEITOS DA OXIDAÇÃO POR PERSULFATO NAS PROPRIEDADES DO LATOSSOLO VERMELHO
}

\author{
Mariana Favero $^{1 *}$; Juliana G. de Freitas ${ }^{1}$; Sheila A. C. Furquim ${ }^{1}$; Jennifer Sousa ${ }^{1} \&$ \\ Larissa M. Godke ${ }^{1}$
}

\section{Resumo}

O persulfato é um oxidante de uso crescente na oxidação química in situ, no entanto, os impactos deste nas propriedades dos solos, e consequentemente no transporte de solutos e na eficiência da remediação, são pouco conhecidos. Neste sentido, buscou-se avaliar as alterações físicas em um solo tropical resultante da oxidação com persulfato. Para isso, foram realizados experimentos de transporte em sistemas unidimensionais utilizando uma coluna de Latossolo Vermelho indeformado em regime saturado. Foi realizada a injeção do brometo para a obtenção de parâmetros de transporte e da porosidade da coluna. A oxidação com persulfato de potássio (12 $\pm 1 \mathrm{~g} / \mathrm{L})$ foi realizada por 30 dias. Antes da oxidação, a injeção de brometo resultou em dispersividade longitudinal igual a $2,4311 \pm 0,0022 \mathrm{~cm}$ e porosidade efetiva igual 41,99 $\pm 1,52 \%$. Na oxidação, foi observada queda abrupta do $\mathrm{pH}(3,0-3,5)$ e liberação de Al (III). A condutividade hidráulica aumentou consideravelmente, provavelmente devido à formação de caminhos preferenciais na coluna de solo. Verificou-se, assim, que a oxidação com persulfato resultou em alterações nas condições do solo ( $\mathrm{pH}$ e Eh) e na dissolução de minerais, o que afeta a estrutura do solo, e como consequência, o transporte de solutos no mesmo.

\begin{abstract}
Persulfate is being used increasingly for in situ chemical oxidation, however, the oxidation impact in soil properties, and therefore in solutes transport and remediation efficiency still lacks understanding. Thus, this work sought to evaluate the changes in physical properties of a tropical soil resulting from potassium persulfate oxidation. One-dimensional laboratory experiments were performed using an undisturbed column of an Oxisol in saturated regime. Transport parameters and the porosity of the column were obtained through the injection of bromide. Oxidation using potassium persulfate $(12 \pm 1 \mathrm{~g} / \mathrm{L})$ was performed for 30 days. Before oxidation, the longitudinal dispersivity was $2,4311 \pm 0,0022 \mathrm{~cm}$ and the effective porosity of the column was $41,99 \pm 1,52 \%$. The injection of persulfate resulted in an abrupt decrease of $\mathrm{pH}(3-3,5)$ and the release of Al(III). The hydraulic conductivity increased considerably, probably due to the generation of preferential paths in the soil
\end{abstract}

${ }^{1}$ Afiliação: UNIFESP - Campus Diadema, Rua São Nicolau, 210, Diadema-SP, Fone: 11 3319-3584, fav.mariana@gmail.com* 
column. Therefore, it was verified that persulfate oxidation resulted in changes in soils conditions ( $\mathrm{pH}$ and Eh) and mineral dissolution, which affects the soil structure, and consequently, solutes transport.

Palavras-Chave - Oxidação, Solos Tropicais, Modelo 1-D

\section{1 - INTRODUÇÃO}

A oxidação química in situ (ISCO, do inglês in situ chemical oxidation) tem sido vista com grande interesse devido à sua eficiência e custo-benefício na remediação de solos e águas subterrâneas contaminados por diversos compostos orgânicos (BACIOCCHI et al., 2014; KREMBS et al., 2010; SEOL; ZHANG; SCHWARTZ, 2003). Esta técnica envolve a distribuição de oxidantes químicos em subsuperfície com o propósito de transformar os contaminantes em espécies menos danosas, reduzindo assim os riscos à saúde pública e qualidade ambiental.Os oxidantes mais comumente utilizados na ISCO são: permanganato $\left(\mathrm{MnO}_{4}{ }^{-}\right)$, peróxido de hidrogênio $\left(\mathrm{H}_{2} \mathrm{O}_{2}\right)$, ozônio $\left(\mathrm{O}_{3}\right)$ e persulfato $\left(\mathrm{S}_{2} \mathrm{O}_{8}{ }^{2-}\right)$ (HULING; PIVETZ, 2006).

Este último, porém, tem sido visto com maior interesse desde 2000-2002, por superar limitações apresentadas por outros oxidantes (BACIOCCHI, 2013; SIEGRIST; CRIMI; SIMPKIN,

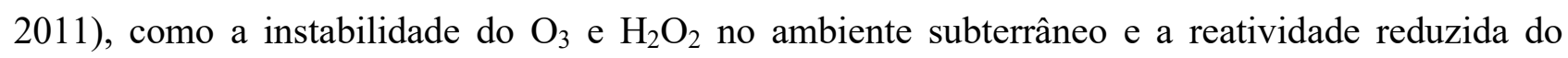
$\mathrm{MnO}_{4}{ }^{-}$com alguns compostos (DENG et al., 2014; FELIX-NAVARRO et al., 2007; LIAO et al., 2014; LUO, 2014; OH; SHIN, 2014; WANG, C. W.; LIANG, 2014; WANG, Z.; DENG; YANG, 2014; ZHAO et al., 2014).

O entendimento dos efeitos da oxidação por persulfato no transporte de solutos do solo, no entanto, ainda é bastante escasso. As informações disponíveis indicam algumas possibilidades dos impactos desse oxidante. Por se tratar de uma abordagem relativamente nova dentro da ISCO, Siegrist et al. (2011) apontam que a mobilidade de metais devido a oxidação por persulfato ainda não foi estudada. No entanto, o forte potencial de oxi-redução do ânion persulfato $\left(E^{0}=2,1 \mathrm{~V}\right)$ e dos radicais sulfatos $\left(\mathrm{E}^{0}=2,6 \mathrm{~V}\right)$ indicam a possibilidade dessas espécies de oxidar diretamente metais e minerais. A capacidade de dessorção de contaminantes pela oxidação por persulfato, por sua vez, tem sido reportada em alguns estudos (CUYPERS et al., 2000; WOODS, 2008). Particularidades do solo local, como a forma da matéria orgânica natural, devem ser consideradas, por terem grande influência no processo de dessorção.

Solos tropicais possuem propriedades físicas, químicas e geotécnicas diferentes dos solos comumente encontrados em regiões temperadas (OSTERREICHER-CUNHA et al., 2007). São, em 
geral, fortemente lixiviados, com baixos teores de bases, baixos a médios teores de sílica e altos teores de ferro e alumínio, havendo consequentemente formação de caulinita (argilomineral 1:1) e/ou óxidos de ferro e alumínio (BIRKELAND, 1984; FANNING; FANNING, 1989).

Estudos envolvendo a oxidação por persulfato em solos brasileiros restringem-se a ensaios em batelada para avaliar a degradação de compostos orgânicos em solos de diferentes texturas, com predominância dos solos arenosos (CIRIACO, 2013; FERNANDES; CHIAVONE FILHO, 2014; GRACIANO et al., 2012; RODRIGUEZ; OLIVEIRA; NASCIMENTO, 2012; SILVA et al., 2015). Assim, a fim de preencher as lacunas existentes, este trabalho teve como objetivo avaliar como o processo de oxidação por persulfato altera as propriedades do Latossolo Vermelho e avaliar, como consequência, a interferência desse processo no transporte de solutos. Para isso foi utilizada uma coluna indeformada de Latossolo Vermelho (LV) em regime saturado como modelo de transporte 1-D.

\section{2 - MATERIAIS E MÉTODOS}

\section{1 - Coleta do solo e montagem do arranjo experimental}

Uma amostra indeformada de Latossolo Vermelho (LV) foi coletada na cidade de PiracicabaSP, na Rodovia Luiz de Queiroz, km 155 (2243’31,7’S - 47³5'9,1” W). Para isso, foi utilizada uma coluna cilíndrica de aço inox (20 cm de altura; $10 \mathrm{~cm}$ de diâmetro) e um amostrador a percussão, de aço e alumínio, desenvolvido para este uso. A coleta foi realizada no horizonte $\mathrm{B}_{\mathrm{w}}$ do LV. Foi feito um corte até a profundidade deste horizonte, a superfície limpa e aplainada para posicionamento da coluna. O solo foi coletado por meio de golpes com um martelo de borracha na haste do amostrador posicionado acima da coluna de aço. Uma vez cravada, foi feito um recorte em volta do solo e retirada a coluna.

Em laboratório, os espaços vazios, sem solo, da coluna foram preenchidos por areia e esfera de vidro $(1 \mathrm{~mm}<\mathrm{d}<3 \mathrm{~mm})$. Nas extremidades foi acrescentada lã de vidro para retenção de material argiloso. A coluna foi fechada com tampa de Teflon ${ }^{\circledR}$ com canais para direcionamento de mangueiras. O arranjo experimental foi montado de maneira adaptada ao descrito por Oliveira (2015). Após a montagem, foi feita a injeção de gás carbônico na coluna a 0,5 psi por 15 minutos, a fim de evitar a presença de ar nos poros e garantir completa saturação d'água (LEWIS; SJOSTROM, 2010). A saturação foi realizada de maneira ascendente com bomba peristáltica a vazão de $9 \mathrm{ml} / \mathrm{h}$ com água sintética ( $\mathrm{pH}$ 5,6-5,9; condutividade elétrica 17-19 $\mu \mathrm{S} / \mathrm{cm}$ ) previamente desaerada. 


\section{2 - Ensaios de transporte}

Dois ensaios de transporte foram realizados consecutivamente na coluna de LV: 1) a injeção de $\mathrm{Br}^{-}$, um traçador ideal, com o qual foram estimados parâmetros da coluna, como a porosidade, a velocidade média e a dispersividade longitudinal; 2) a injeção de persulfato de potássio, em que foi obtida a curva de ruptura do oxidante e avaliada a variação de parâmetros ao longo do tempo ( $\mathrm{pH}$, Eh, condutividade elétrica, condutividade hidráulica, concentração de metais).

Assim, primeiramente, uma solução de água sintética desaerada contendo $30 \pm 1 \mathrm{mg} / \mathrm{L}$ de brometo foi injetada na coluna utilizando bomba peristáltica com fluxo ascendente a uma vazão de $30 \pm 1 \mathrm{~mL} / \mathrm{h}$. A injeção de brometo foi feita por tempo suficiente para que ocorresse estabilização da concentração e então, foi injetada água sintética desaerada até completa saída do brometo. A concentração do brometo foi determinada utilizando um eletrodo seletivo (9635BNWP, Thermo Scientific) acoplado a um medidor multiparâmetros pH/ISE (Orion Star A324, Thermo Scientific). A vazão do efluente e a carga hidráulica foram medidas periodicamente. O ensaio foi feito em duplicata, sendo que a segunda injeção de brometo só foi realizada após completa lavagem da coluna.

Obtida a curva de ruptura para o brometo $\left(\mathrm{C} / \mathrm{C}_{0}\right.$ versus nvp - número de volume de poros), foi feito ajuste dos dados experimentais à solução analítica da equação de transporte de massa em 1-D, proposta por Ogata e Banks (1961), assumindo $\mathrm{R}=1$. O ajuste foi feito utilizando a ferramenta Solver do Excel para calcular a velocidade (vx) e a dispersão longitudinal (Dx) de maneira que a diferença entre os dados experimentais e os dados modelados fosse mínima. Com a velocidade determinada, a porosidade pôde ser estimada pela Lei de Darcy (FETTER, 2001). A partir desses parâmetros, utilizando o coeficiente de difusão molecular $\left(\mathrm{D}_{\mathrm{m}}\right)$ para o brometo obtido em Cussler (2007), foi calculada a dispersividade longitudinal $\left(\alpha_{\mathrm{L}}\right)$ (Equação 1):

$$
D_{\mathrm{x}}=\mathrm{D}_{\mathrm{L}}=\alpha_{\mathrm{L}} \mathrm{V}_{\mathrm{x}}+\tau \mathrm{D}_{\mathrm{m}}
$$

Em que $\tau$ é a tortuosidade do meio.

Para a oxidação da coluna indeformada de LV, foi utilizada solução sintética desaerada contendo $12 \pm 1 \mathrm{~g} / \mathrm{L}$ de persulfato. A injeção foi realizada por 30 dias à vazão $15 \mathrm{~mL} / \mathrm{h}$. Após esse período a coluna foi lavada com água sintética até completa saída do persulfato. As amostras, para a determinação da concentração de persulfato e de metais, foram coletadas em menores intervalos nas primeiras 100 horas de ensaio e em menor frequência no período restante.

A concentração do persulfato foi determinada por meio de espectrofotômetro de UV-vis (Genesys 10S UV-vis, Thermo Scientific) utilizando método proposto por Huang et al. (2002). Para 
os metais foi analisada a concentração de Al (III) e Fe (II). Para o primeiro, foi utilizado método adaptado de Cantarella et al. (2001). O Fe (II) foi determinado por uma adaptação dos métodos de Jeffery et al. (1889 - p. 691) e SABESP (2001).

$\mathrm{O}$ pH foi registrado periodicamente utilizando medidor multiparâmetros $\mathrm{pH} / \mathrm{ISE}$ (eletrodo triodo combinado 9107BNMD, medidor Orion Star A324, Thermo Scientific). Foram registrados, na mesma frequência em que foram coletadas as amostras de persulfato, a condutividade elétrica utilizando medidor portátil (LAQUAtwin, Horiba) e o Eh utilizando medidor pH/ORP \& EC/TDS/ $\mathrm{NaCl}$ (HI 2550, Hanna Instruments). A carga hidráulica foi verificada e registrada pelo menos uma vez por dia.

\section{3 - RESULTADOS E DISCUSSÃO}

\section{1 - Injeção de traçador conservativo}

A concentração de $\mathrm{Br}^{-}$do efluente se igualou à concentração de entrada em 50,37 h ou 2,25 nvp na injeção 1 e em 48,3 h ou 2,28 nvp para a injeção 2. Nos dois casos, o Br- não apresentou retardamento na coluna de LV. Os parâmetros estimados após o ajuste dos dados experimentais à solução analítica proposta por Ogata e Banks (1961) são apresentados a seguir (Tabela 1):

Tabela 1. Parâmetros estimados a partir das injeções de $\operatorname{Br}^{-}(30 \pm 1 \mathrm{mg} / \mathrm{L})$ na coluna de LV

\begin{tabular}{|c|c|c|c|c|}
\cline { 2 - 5 } \multicolumn{1}{c|}{} & \multicolumn{4}{c|}{ Parâmetros } \\
\cline { 2 - 5 } \multicolumn{1}{c|}{} & $\mathbf{v}_{\mathbf{x}}(\mathbf{c m} / \mathbf{m i n})$ & $\mathbf{D}_{\mathbf{L}}\left(\mathbf{c m}^{\mathbf{2}} / \mathbf{m i n}\right)$ & $\boldsymbol{\theta} \mathbf{( \% )}$ & $\left.\boldsymbol{\alpha}_{\mathbf{L}} \mathbf{( c m}\right)$ \\
\hline Injeção 1 & 0,0149 & 0,0363 & 43,06 & 2,4326 \\
\hline Injeção 2 & 0,0158 & 0,0383 & 40,91 & 2,4295 \\
\hline Média & $0,0153 \pm 0,0006$ & $0,0373 \pm 0,0014$ & $41,99 \pm 1,52$ & $2,4311 \pm 0,0022$ \\
\hline
\end{tabular}

A porosidade obtida pelo ensaio de transporte pode ser comparada à porosidade do solo $(61,68 \pm 2,17 \%)$ medida por Oliveira (2015). No momento da coleta, esta coluna apresentou compactação de 20,98 \%. Porém, a porosidade medida pelo ensaio de transporte foi $30 \%$ menor que a porosidade total. Isso acontece, porque a porosidade estimada pelo ensaio de transporte é a porosidade efetiva $\left(\mathrm{n}_{\mathrm{e}}\right)$, inferior à total. A porosidade efetiva é dada pela relação entre o volume de poros interconectados em relação ao volume total do solo, estando mais relacionada ao fluxo de água em um meio que a porosidade total (DAWSON, 2008; SCHWARTZ; ZHANG, 2003).

Considerando que a dispersividade, que é uma característica do meio, tem relação direta com a escala e de maneira aproximada é $1 / 10$ da distância percorrida (GELHAR; WELTY, 1992), a 
dispersividade obtida está dentro da ordem de grandeza esperada, uma vez que a distância de viagem é de $20 \mathrm{~cm}$.

A partir dos dados de carga hidráulica, foi calculada a condutividade hidráulica $(K)$ durante a injeção de brometo, utilizando a Lei de Darcy. Como resultado, obteve-se uma variação na condutividade hidráulica de 6,31E-04 a 1,11E-03 cm/s.

\section{1 - Oxidação}

O persulfato estabilizou a partir das $100 \mathrm{~h}$ de ensaio em concentração equivalente a $0,80 \mathrm{em}$ relação à concentração inicial. Esse resultado é coerente quando considerada a persistência do persulfato em meio ambiente subterrâneo, conforme observado por Sra et al. (2010). Eles notaram que, diferentemente do permanganato, o persulfato não possui uma demanda natural finita, sendo continuamente consumido em diferentes materiais de aquíferos.

Os resultados de Eh e condutividade elétrica seguiram a mesma tendência observada na curva de ruptura do persulfato, com estabilização após as $100 \mathrm{~h}$ de ensaio. $\mathrm{O}$ Eh inicial da coluna foi igual a $96,95 \mathrm{mV}$ e o valor médio atingido após a estabilização foi iguala $658,81 \pm 17,31 \mathrm{mV}$, indicando a formação de um ambiente oxidante devido a presença de persulfato em excesso. A condutividade elétrica, por sua vez, atingiu a média de $9,74 \pm 0,54 \mathrm{mS} / \mathrm{cm}$. Tal valor tem relação com a liberação de íons no efluente, como o Al (III), porém sem relação com a concentração de Fe (II), que permaneceu nula durante a injeção de persulfato.

A concentração de Al (III) teve aumento abrupto de maneira concomitante com a redução também intensa do $\mathrm{pH}$, que permaneceu entre 3,5-3,0 após as $100 \mathrm{~h}$ de injeção. Observou-se um pico de liberação de Al (III) em 80 h de ensaio, com concentração 299,77 mg/L, e posterior estabilização da concentração em $216,07 \pm 13,80 \mathrm{mg} / \mathrm{L}$. O processo observado pode ser comparado com a intemperização de solos tropicais, quando o pH é inferior a 4,5, o que ocorre na presença de ácidos orgânicos. Nessa situação de pH extremo, o alumínio fica muito mais solúvel que a sílica ou ferro e é, então, lixiviado (BREEMEN; BUURMAN, 2002).

A condutividade hidráulica atingiu o valor máximo de $5,19 \mathrm{E}-03 \mathrm{~cm} / \mathrm{s}$. Acredita-se que a dissolução de materiais dos minerais e, consequente formação de caminhos preferenciais na coluna de solo, ocasionou no aumento da condutividade hidráulica do solo. 


\section{4 - AGRADECIMENTOS}

Os autores agradecem o apoio recebido da FAPESP (Fundação de Amparo à Pesquisa do Estado de São Paulo) por meio do auxílio Jovem Pesquisador (2011/12158-2)e de bolsa de mestrado (2015/11199-8).

\section{5 - REFERÊNCIAS}

BACIOCCHI, R. Principles, Developments and Design Criteria of In Situ Chemical Oxidation. Water, Air, \& Soil Pollution, v. 224, n. 12, p. 1-11, 2013.

BACIOCCHI, R. et al. Development of technical guidelines for the application of in-situ chemical oxidation to groundwater remediation. Journal of Cleaner Production, v. 77, p. 47-55, 2014.

BIRKELAND, P. W. Soils and Geomorphology. Oxford: Oxford University Press, 1984.

BREEMEN, N.; BUURMAN, P. Soil Formation. 2 ${ }^{\mathrm{a}}$ ed. Dordrecht: Kluwer Academic Publishers, 2002.

CANTARELlA, H. et al. Determinação de Alumínio, Cálcio e Magnésio Trocáveis em Extrato de Cloreto de Potássio. In: Análise Química para Avaliação da Fertilidade de Solos Tropicais. Campinas: Instituto Agronômico de Campinas, 2001.

CIRIACO, M. F. Degradação de Hidrocarbonetos Policíclicos Aromáticos em Solos Arenosos Empregando Processos Oxidativos. 2013. Dissertação (Mestrado em Química). Universidade de São Paulo, Sao Paulo, Brazil, 2013.

CUYPERS, C. et al. Rapid persulfate oxidation predicts PAH bioavailability in soils and sediments. Environmental Science \& Technology, v. 34, n. 10, p. 2057-2063, 2000.

DAWSON, A. Water in Road Structures: Movement Drainage and Effects. Nottingham: Springer 2008.

DENG, D. Y. et al. Impact of activation methods on persulfate oxidation of methyl tert-butyl ether. Journal of Hazardous Materials, v. 264, p. 521-528, 2014.

FANNING, D. S.; FANNING, M. C. B. Soil: Morphology, genesis and Classification. Nova York: Wiley, 1989.

FELIX-NAVARRO, R. M. et al. Kinetics of the degradation of 1,4-dioxane using persulfate. Journal of the Mexican Chemical Society, v. 51, n. 2, p. 67-71, 2007.

FERNANDES, H. G.; CHIAVONE FILHO, O. Avaliação Técnica da Remediação de Solo Utilizando Persulfato/Peróxido de Hidrogênio. Revista Eletrônica do Mestrado em Petróleo e Gás, v. 2, n. 2, p. 21-30, 2014.

FETTER, C. W. Applied Hydrogeology. New Jersey: Prentice-Hall, 2001. 
GELHAR, L. W.; WELTY, C. A Critical Review of Data on Field-Scale Dispersion in Aquifers. Water Resources Research, v. 28, n. 7, p. 1955-1974, 1992.

GRACIANO, V. A. et al. Remediação de um Solo Contaminado com Petróleo por Oxidação Química. Revista Brasileira de Ciência do Solo, v. 36, n. 1, p. 1656-1660, 2012.

HUANG, K.-C.; COUTTENYE, R. A.; HOAG, G. E. Kinetics of heat-assisted persulfate oxidation of methyl tert-butyl ether (MTBE). Chemosphere, n. 49, p. 413-420, 2002.

HUlinG, S. G.; PIVETZ, B. E. EPA Engineering Issue: In-Situ Chemical Oxidation. 2006. Disponível em: $<$ http://www.epa.gov/ada/gw/pdfs/insituchemicaloxidation_engineering_issue.pdf $>$. Acesso em: 22 set. 2014.

JEFFERY, G. H. et al. Vogel's: Textbook of Quantitative Chemical Analysis. 5 ed. Essex: Longman Scientific \& Technical, 1989.

KREMBS, F. J. et al. ISCO for Groundwater Remediation: Analysis of Field Applications and Performance. Ground Water Monitoring and Remediation, v. 30, n. 4, p. 42-53, 2010.

LEWIS, J.; SJOSTROM, J. Optimizing the experimental design of soil columns in saturated and unsaturated transport experiments. Journal of Contaminant Hydrology, v. 115, n. 1-4, p. 1-13, 2010.

LIAO, X. Y. et al. Identification of persulfate oxidation products of polycyclic aromatic hydrocarbon during remediation of contaminated soil. Journal of Hazardous Materials, v. 276, p. 26-34, 2014.

LUO, Q. S. Oxidative treatment of aqueous monochlorobenzene with thermally-activated persulfate. Frontiers of Environmental Science \& Engineering, v. 8, n. 2, p. 188-194, 2014.

OGATA, A.; BANKS, R. B. A solution of the differential equation of longitudinal dispersion in porous media. In: Geological Survey Professional Paper 411-A. Washington: United States Government Printint Office, 1961.

OH, S. Y.; SHIN, D. S. Treatment of Diesel-Contaminated Soil by Fenton and Persulfate Oxidation with Zero-Valent Iron. Soil \& Sediment Contamination, v. 23, n. 2, p. 180-193, 2014.

OLIVEIRA, F. C. Avaliação da Interação entre o Persulfato de Potássio com Solos Brasileiros para a Utilização da Tecnologia de Remediação por Oxidação Química In Situ. 2015. Dissertação de Mestrado em Engenharia. Engenharia Química, Universidade de São Paulo, São Paulo, 2015.

OSTERREICHER-CUNHA, P. et al. Study of biodegradation processes of BTEX-ethanol mixture in tropical soil. Water Air and Soil Pollution, v. 181, n. 1-4, p. 303-317, 2007.

RODRIGUEZ, C. P.; OLIVEIRA, S. M. B.; NASCIMENTO, C. A. O. A influência das Características dos Solos na Remediação de Locais Contaminados através de Processos Oxidativos Avançados com Persulfato e Reagente de Fenton. Águas Subterrâneas, v. 26, n. 1, p. 131-141, 2012. 
SCHWARTZ, F. W.; ZHANG, H. Fundamentals of Groundwater. New York: John Wiley \& Sons, 2003.

SEOL, Y.; ZHANG, H.; SCHWARTZ, F. W. A review of in situ chemical oxidation and heterogeneity. Environmental \& Engineering Geoscience, v. 9, n. 1, p. 37-49, 2003.

SIEGRIST, R. L.; CRIMI, M.; SIMPKIN, T. J. In Situ Chemical Oxidation for Groundwater Remediation. New York, USA: Springer Science+Business Media, 2011.

SILVA, C. K. O. et al. Tratamento de fenantreno utilizando persulfato de sódio ativado com ferro endógeno presente em solos arenosos. In: XXXV Congresso Brasileiro de Ciência do Solo, 2015, Natal. 2015.

SRA, K. S.; THOMSON, N. R.; BARKER, J. F. Persistence of Persulfate in Uncontaminated Aquifer Materials. Environmental Science \& Technology, v. 44, n. 8, p. 3098-3104, 2010.

WANG, C. W.; LIANG, C. J. Oxidative degradation of TMAH solution with UV persulfate activation. Chemical Engineering Journal, v. 254, p. 472-478, 2014.

WANG, Z.; DENG, D. Y.; YANG, L. L. Degradation of dimethyl phthalate in solutions and soil slurries by persulfate at ambient temperature. Journal of Hazardous Materials, v. 271, p. 202-209, 2014.

WOODS, L. M. In situ remediation induced changes in subsurface properties and trichloroethene partitioning behavior. 2008. Thesis (Masters). Division of Environmental Science and Engineering, Colorado School of Mines, Golden, 2008.

ZHAO, L. et al. Degradation of 1,4-dioxane in water with heat- and Fe2+-activated persulfate oxidation. Environmental Science and Pollution Research, v. 21, n. 12, p. 7457-7465, 2014. 